\title{
The Mechanism and Origin of Enantioselectivity in the Rhodium-Catalyzed Asymmetric Ring-Opening Reactions of Oxabicyclic Alkenes with Organoboronic Acids: A DFT Investigation
}

\author{
Austin Pounder, William Tam and Leanne D. Chen* \\ Guelph-Waterloo Centre for Graduate Work in Chemistry and Biochemistry, Department of Chemistry, University of \\ Guelph, Guelph, Ontario, Canada N1G 2W1
}

\section{Table of Contents}

Free Energy Diagrams.

3D structures and energies for the ARO of OA and phenylboronic acid .

Free energies, total electronic energies, and the imaginary frequencies for optimized geometries 


\section{Free Energy Diagrams}

Figure S1. Potential energy profile of the SMD solvation model for the Rh-Catalyzed ARO of OA with phenylboronic acid $\mathbf{8 b}$ in THF/water $(10: 1 \mathrm{v} / \mathrm{v}$ ), as evaluated by DFT. Calculated Gibbs free energies (in $\mathrm{kcal} / \mathrm{mol}$; (B3LYP $25,26 /$ def2SVP27/SMD $29 / /$ B3LYP $25,26 /$ def2TZVP $\left.27 / S^{29}{ }^{29}\right), \mathrm{T}=298.15 \mathrm{~K}$ ) are with respect to separated reactants TMRh-metal $+\mathrm{H}_{2} \mathrm{O}+\mathbf{O A}$. Hydrogen atoms in geometries shown (except those directly participating) have been omitted for clarity.

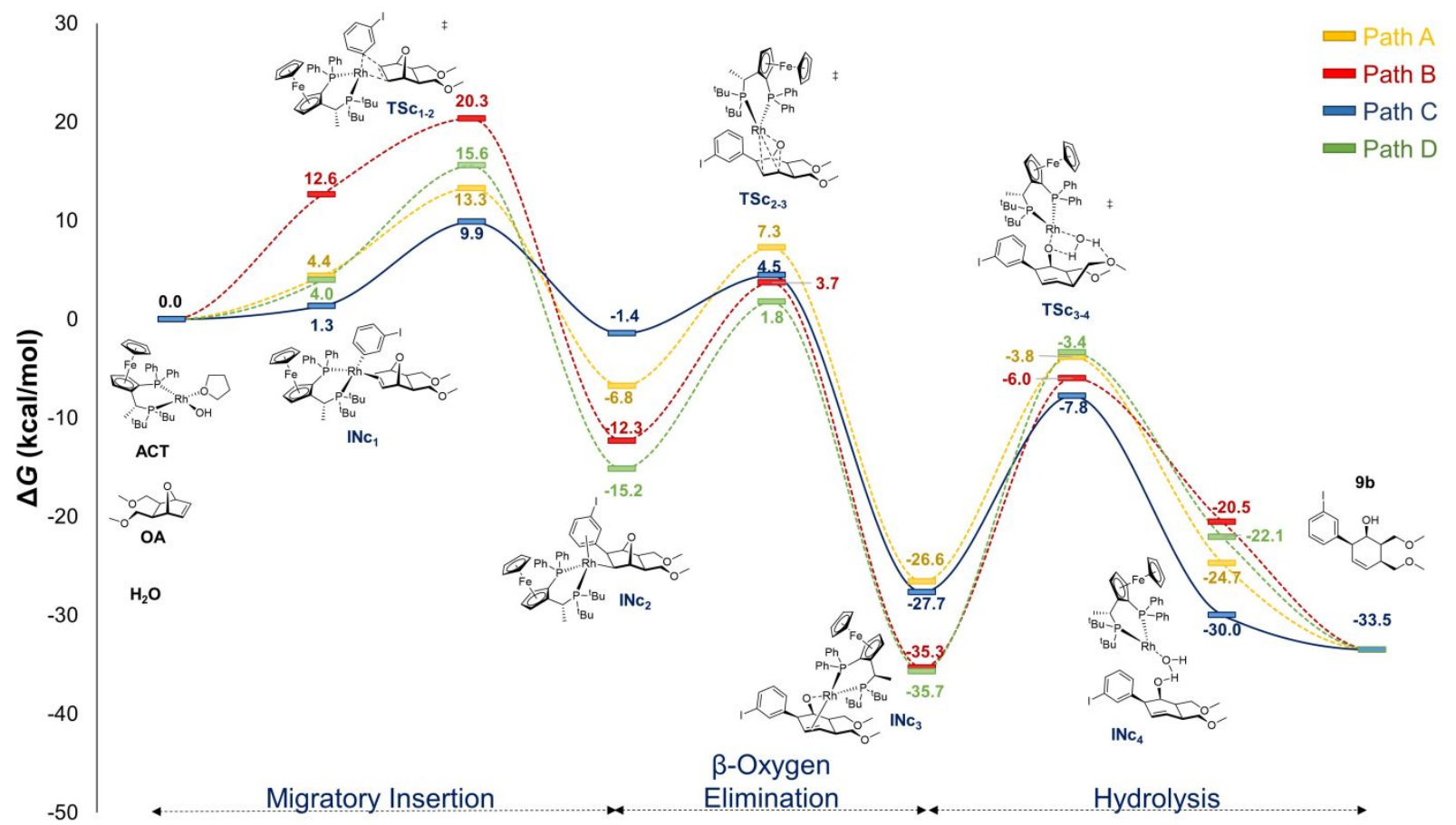


Figure S2. Potential energy profile of the SMD solvation model for the Rh-Catalyzed ARO of OA with $p$ methoxyphenylboronic acid 8c in THF/water $(10: 1 \mathrm{v} / \mathrm{v})$, as evaluated by DFT. Calculated Gibbs free

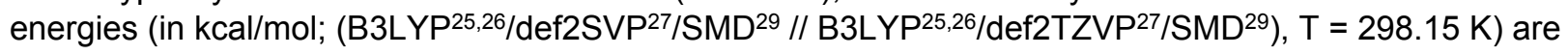
with respect to separated reactants TMRh-paraOMe $+\mathrm{H}_{2} \mathrm{O}+\mathrm{OA}$. Hydrogen atoms in geometries shown (except those directly participating) have been omitted for clarity.

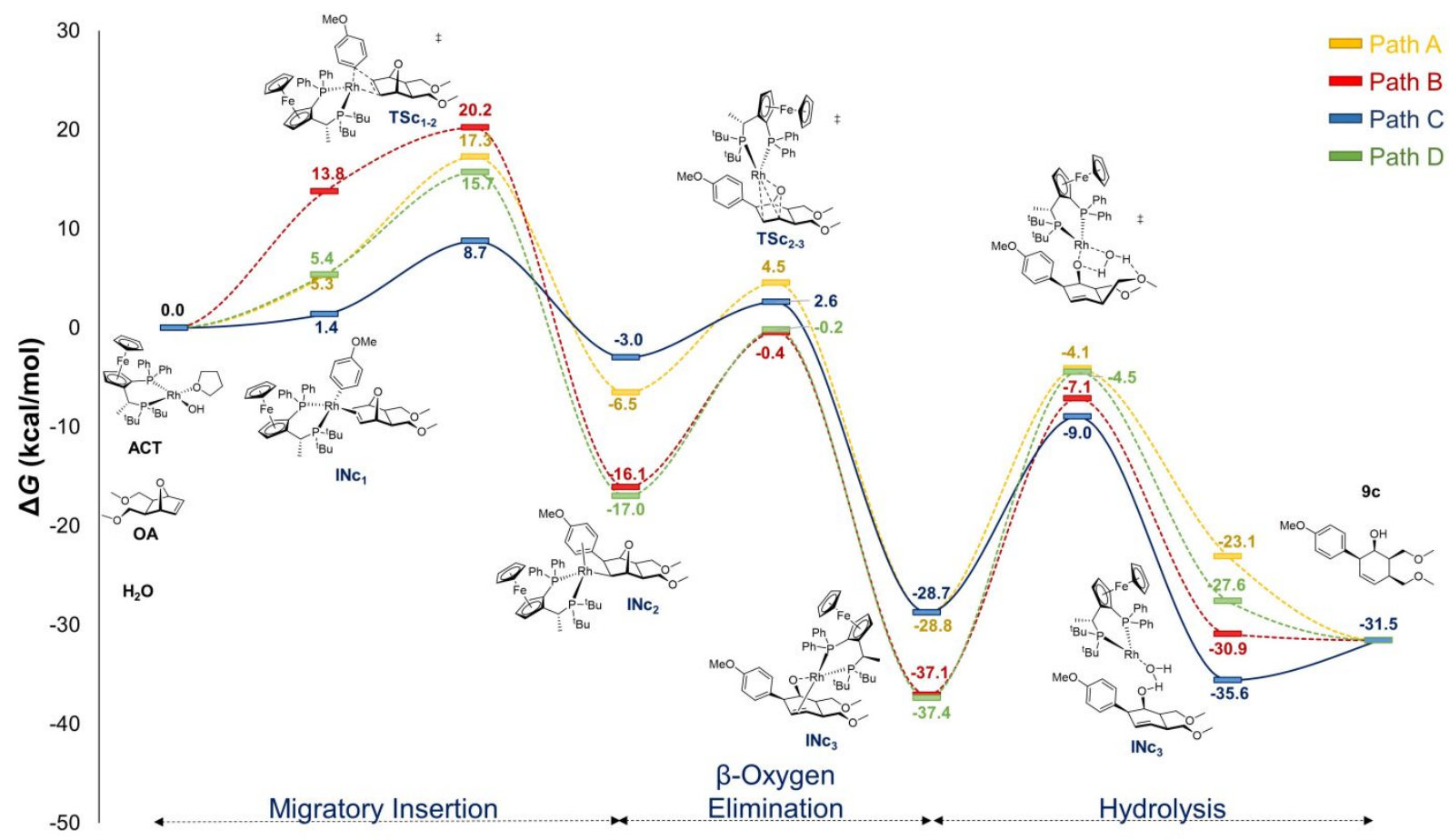


3D structures and energies for the ARO of OA and phenylboronic acid
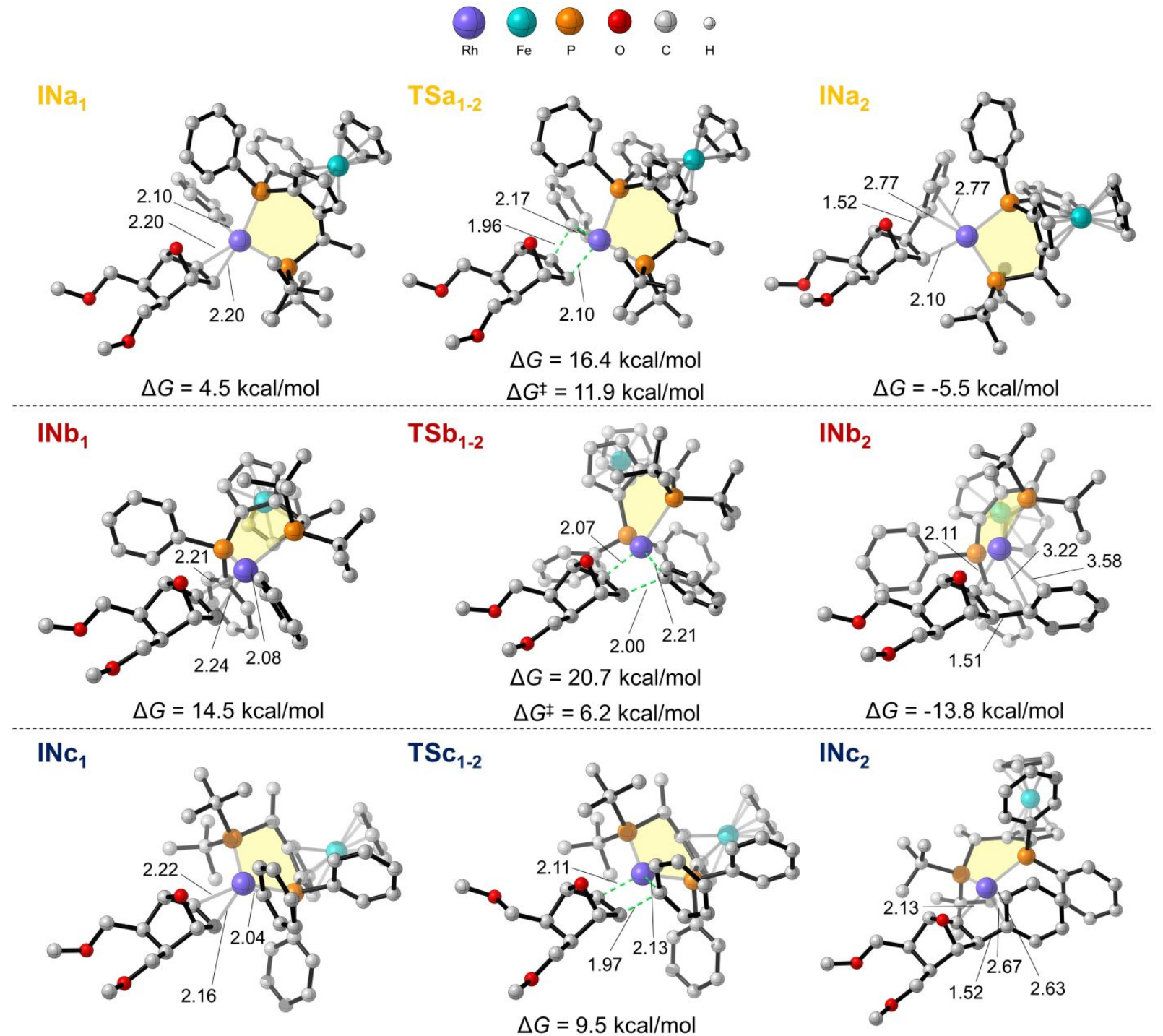

$\mathrm{TSc}_{1-2}$
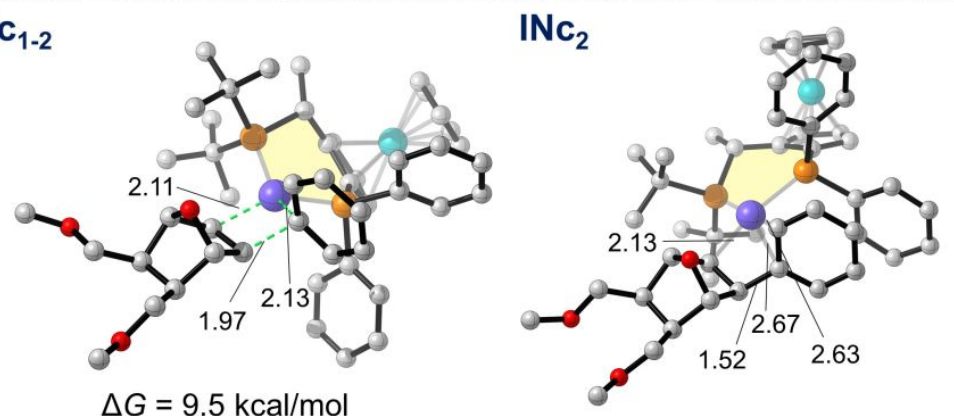

$\Delta G=2.2 \mathrm{kcal} / \mathrm{mol}$

$\Delta G^{\ddagger}=7.3 \mathrm{kcal} / \mathrm{mol}$

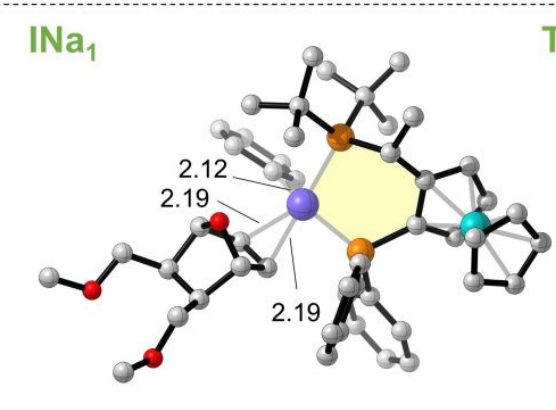

$\Delta G=6.2 \mathrm{kcal} / \mathrm{mol}$

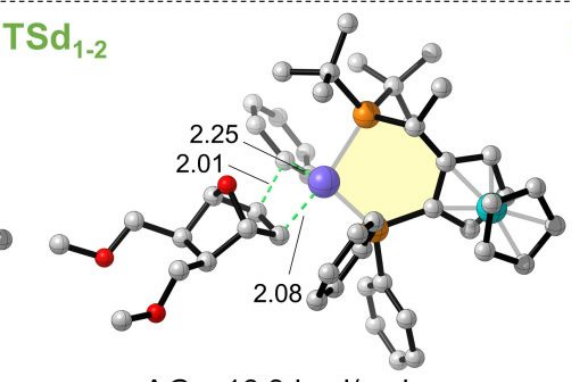

$\Delta G=16.3 \mathrm{kcal} / \mathrm{mol}$

$\Delta G^{\ddagger}=10.2 \mathrm{kcal} / \mathrm{mol}$
$\Delta G=-1.4 \mathrm{kcal} / \mathrm{mol}$

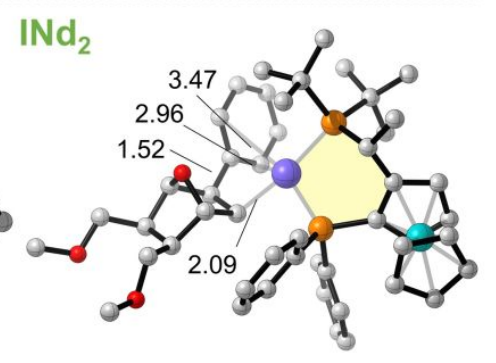

$\Delta G=-16.0 \mathrm{kcal} / \mathrm{mol}$

Figure S3. Optimized geometries for the migratory insertion step in the Rh-catalyzed asymmetric ringopening of OA with phenylboronic acid in THF/water $(10: 1 \mathrm{v} / \mathrm{v})$, as evaluated by DFT calculation (B3LYP ${ }^{25,26 / d e f 2 S V P}{ }^{27} / S_{M D}{ }^{29} / /$ B3LYP $25,26 /$ def2TZVP27/SMD ${ }^{29}$ ). Calculated Gibbs free energies (in $\mathrm{kcal} / \mathrm{mol} ; T=298.15 \mathrm{~K}$ ) are with respect to separated reactants $\mathbf{T M R h}+\mathbf{O A}+\mathbf{H}_{\mathbf{2}} \mathbf{O}$. The dotted lines in the illustration of the transition states represent bonds being formed. Hydrogen atoms in geometries shown (except those directly participating) have been omitted for clarity. The bond lengths are given in $\AA$. 

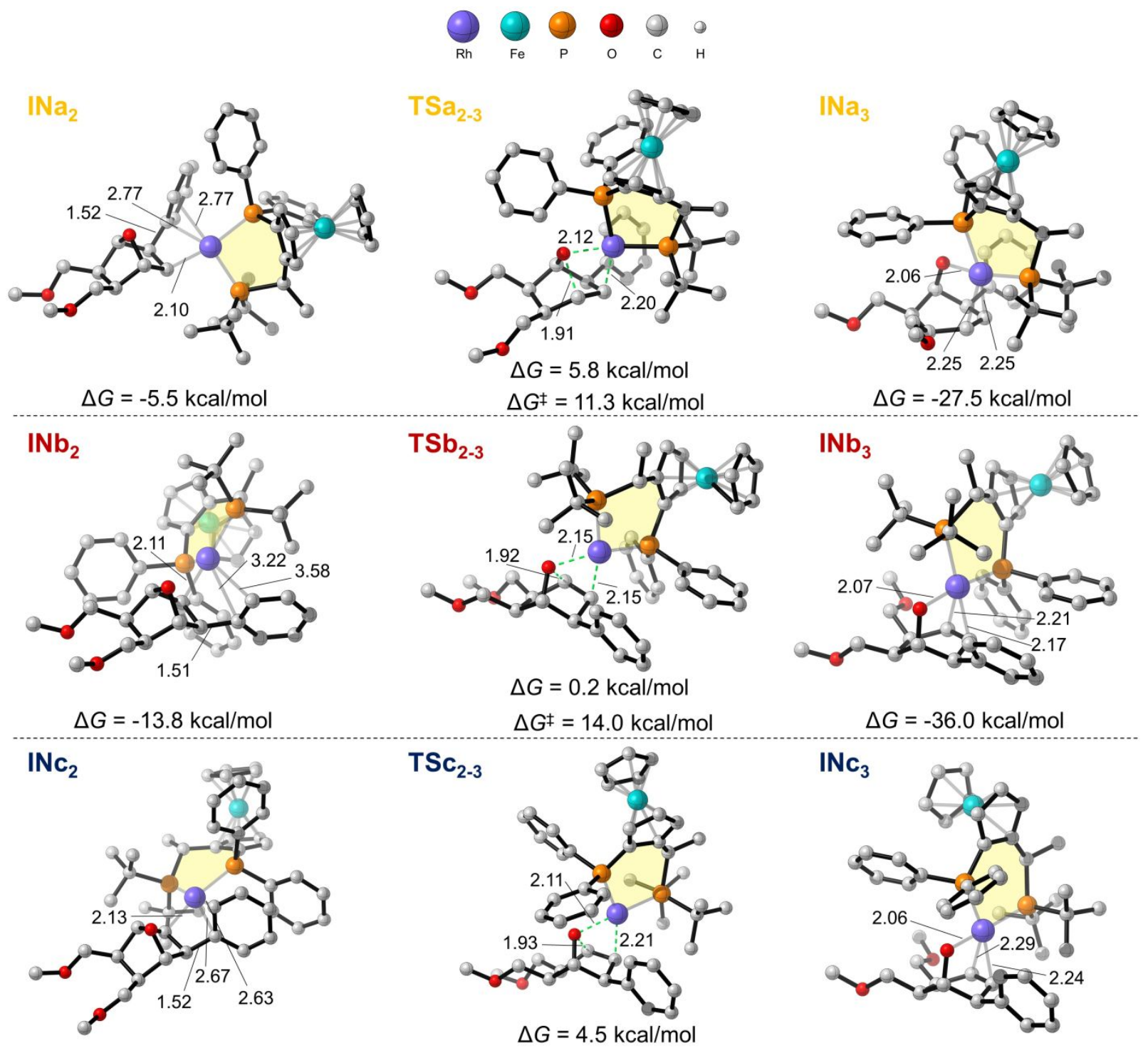

$\Delta G=-1.4 \mathrm{kcal} / \mathrm{mol}$

$\Delta G^{\ddagger}=5.9 \mathrm{kcal} / \mathrm{mol}$

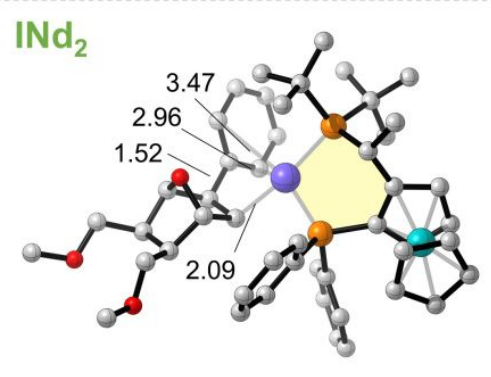

$\Delta G=-16.0 \mathrm{kcal} / \mathrm{mol}$
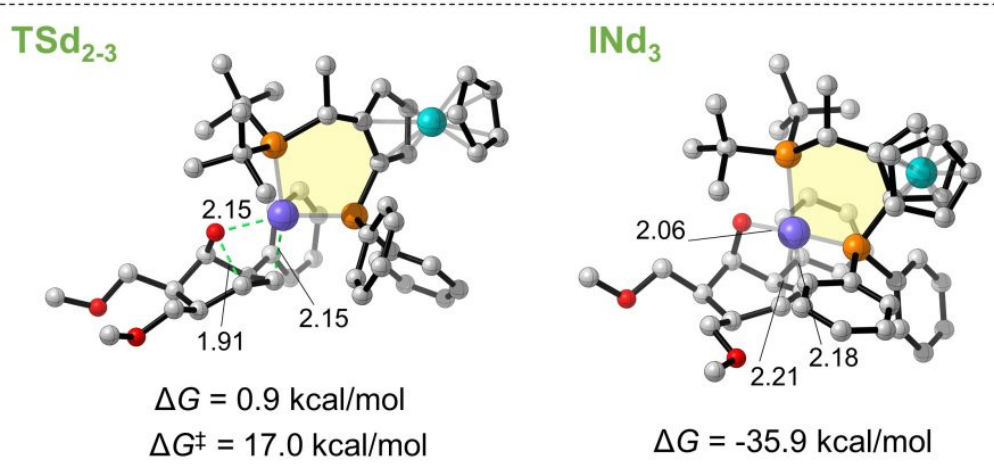

Figure S4. Optimized geometries for the $\beta$-Oxygen Elimination step in the Rh-catalyzed asymmetric ringopening of OA with phenylboronic acid in THF/water $(10: 1 \mathrm{v} / \mathrm{v})$, as evaluated by DFT calculation (B3LYP ${ }^{25,26 / d e f 2 S V P}{ }^{27} / S_{M D}{ }^{29} / /$ B3LYP $25,26 /$ def2TZVP27/SMD 29$)$. Calculated Gibbs free energies (in $\mathrm{kcal} / \mathrm{mol} ; T=298.15 \mathrm{~K}$ ) are with respect to separated reactants TMRh $+\mathbf{O A}+\mathbf{H}_{2} \mathbf{O}$. The dotted lines in the illustration of the transition states represent bonds being formed. Hydrogen atoms in geometries shown (except those directly participating) have been omitted for clarity. The bond lengths are given in $\AA$. 

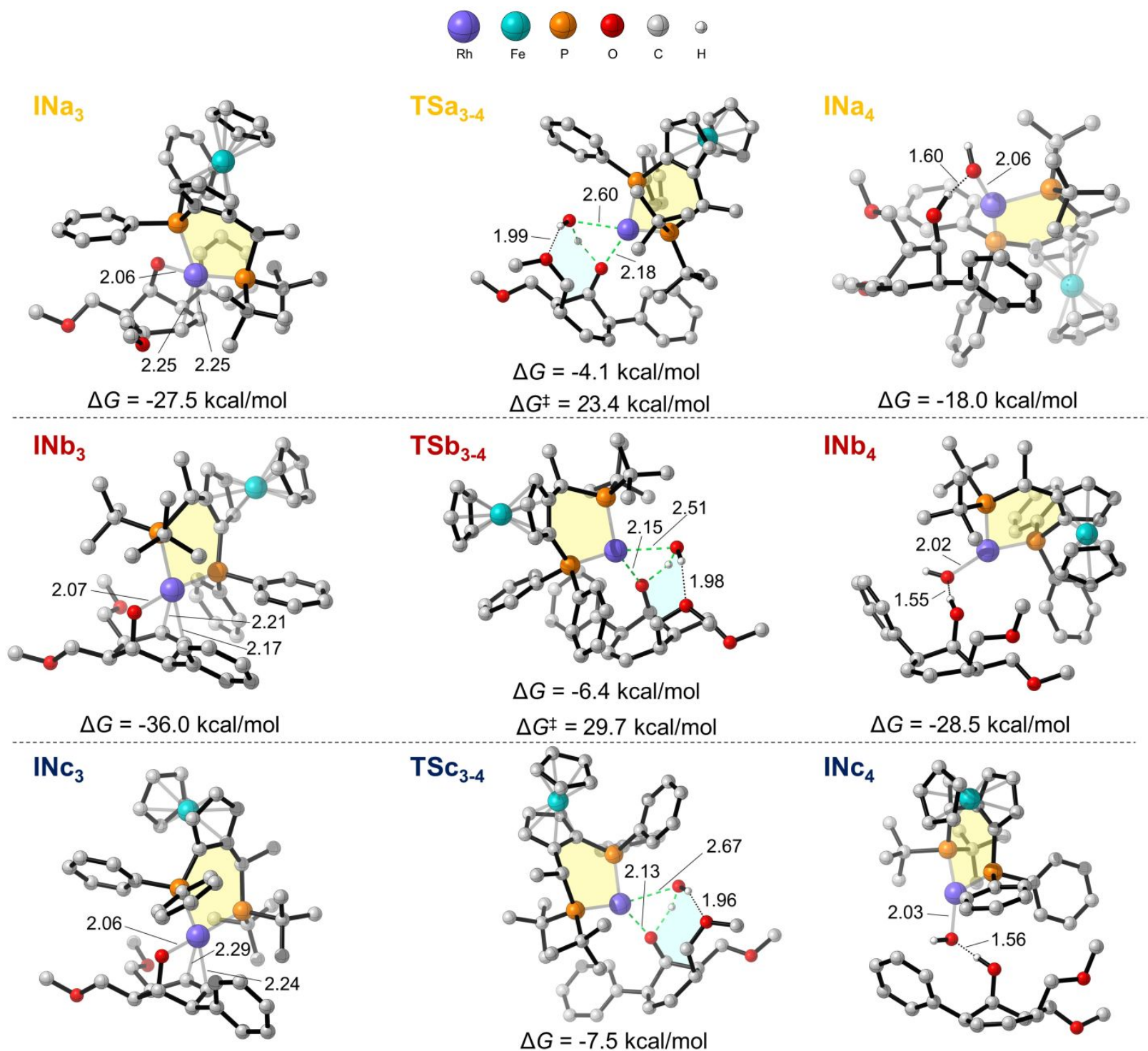

$\Delta G=-28.5 \mathrm{kcal} / \mathrm{mol}$

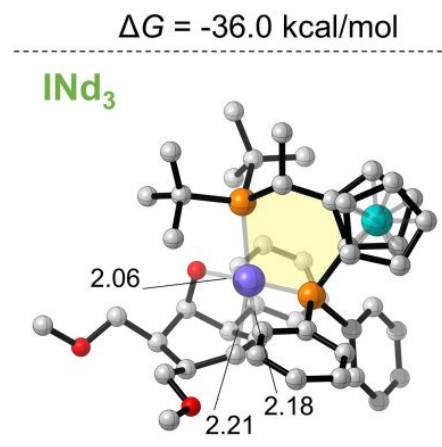

$\Delta G=-35.9 \mathrm{kcal} / \mathrm{mol}$
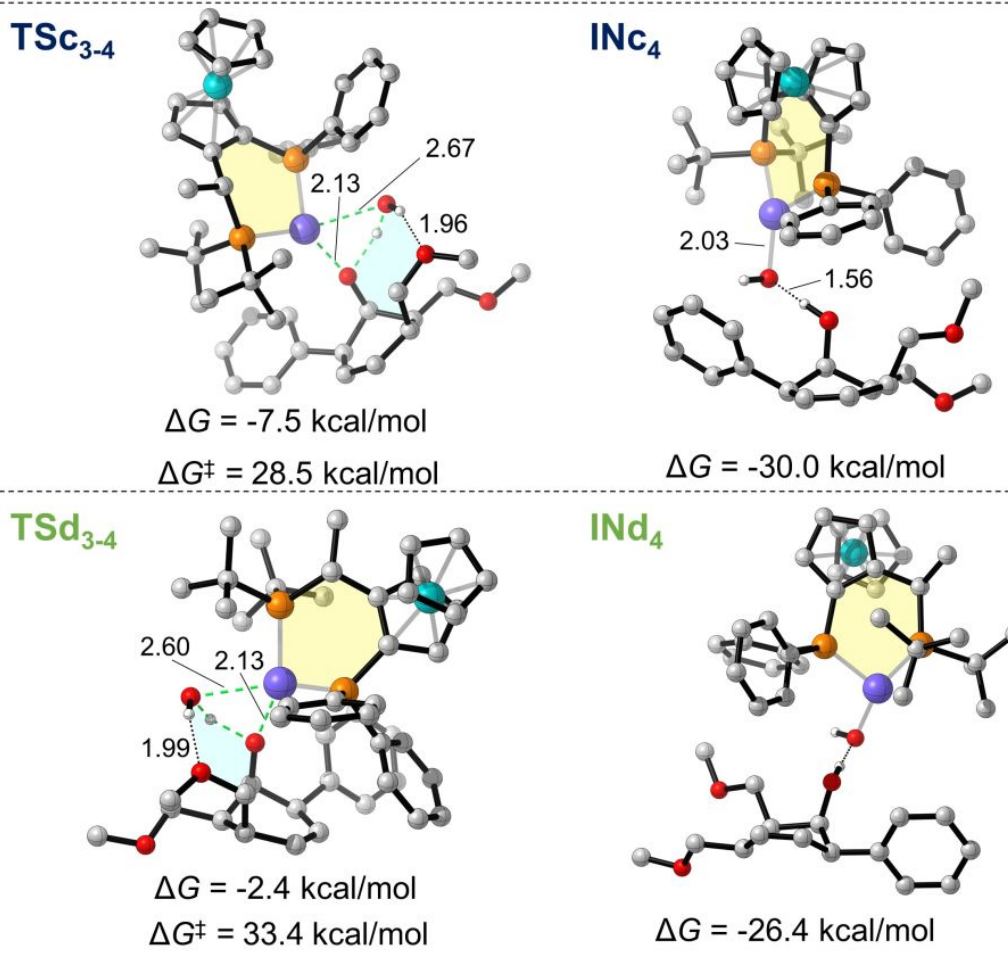

$$
\begin{aligned}
\Delta G & =-7.5 \mathrm{kcal} / \mathrm{mol} \\
\Delta G & =28.5 \mathrm{kcal} / \mathrm{mol} \quad \Delta G=-30.0 \mathrm{kcal} / \mathrm{mol}
\end{aligned}
$$

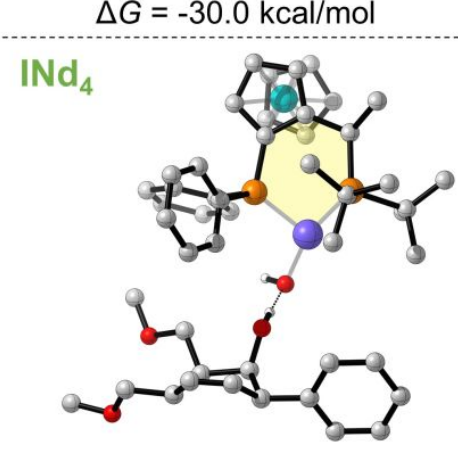

$\Delta G=-26.4 \mathrm{kcal} / \mathrm{mol}$

Figure S5. Optimized geometries for the Hydrolysis step in the Rh-catalyzed asymmetric ring-opening of OA with phenylboronic acid in THF/water $(10: 1 \mathrm{v} / \mathrm{v})$, as evaluated by DFT calculation (B3LYP ${ }^{25,26 / d e f 2 S V P}{ }^{27} / S_{M D}{ }^{29} / / B_{3} L Y P^{25,26} /$ def2TZVP ${ }^{27} / S_{M D}{ }^{29}$ ). Calculated Gibbs free energies (in $\mathrm{kcal} / \mathrm{mol} ; T=298.15 \mathrm{~K}$ ) are with respect to separated reactants $\mathbf{T M R h}+\mathbf{O A}+\mathbf{H}_{2} \mathbf{O}$. The dotted lines in the illustration of the transition states represent bonds being formed. Hydrogen atoms in geometries shown (except those directly participating) have been omitted for clarity. The bond lengths are given in $\AA$. 
Free energies, total electronic energies, and the imaginary frequencies for optimized geometries

Table S1. Free energies (G), total electronic energies $(E)$, and the imaginary frequencies in solvent (tetrahydrofuran/water 10:1, v/v) for the optimized species for the activation of the precatalyst and the ARO reaction of phenylboronic acid $8 \mathbf{a}$ and $\mathbf{O A}$, as evaluated by DFT calculation (B3LYP $25,26 /$ def2SVP $27 / S M D^{29} / / \mathrm{B}^{2} \mathrm{LYP}^{25,26} / \mathrm{def} 2 \mathrm{TZVP}^{27} / \mathrm{SMD}^{29}$ ).<smiles>COCC1C=CC(c2ccccc2)C(O)C1COC</smiles>

\begin{tabular}{|c|c|c|c|}
\hline Species & $G$ (hartree) & $E$ (hartree) & Imaginary Frequency $\left(\mathrm{cm}^{-1}\right)$ \\
\hline OA & -616.314353 & -616.525292154 & - \\
\hline $\mathbf{8 a}$ & -408.359323 & -408.449894133 & - \\
\hline $\mathrm{H}_{2} \mathrm{O}$ & -76.466792 & -76.4698835690 & - \\
\hline Boric acid & -252.597061 & -252.618739679 & - \\
\hline THF & -232.460576 & -232.548572555 & - \\
\hline ACT & -3376.440882 & -3377.02296487 & - \\
\hline ACT-THF & -3608.872155 & -3609.56825185 & - \\
\hline $\mathrm{TM}_{1-2}$ & -3784.760140 & -3785.46248045 & -151.29 \\
\hline $\mathrm{TM}_{2}$ & -3784.767414 & -3785.47060885 & - \\
\hline $\mathrm{TM}_{2-3}$ & -3784.756037 & -3785.45827711 & -253.82 \\
\hline $\mathrm{TM}_{3}$ & 3784.785381 & -3785.48641704 & - \\
\hline TMRh & -3532.223823 & -3532.87807350 & - \\
\hline TMRh-THF & -3764.644396 & -3765.41326665 & - \\
\hline $\mathrm{INa}_{1}$ & -4148.490983 & -4149.38515798 & - \\
\hline $\mathrm{INb}_{1}$ & -4148.475016 & -4149.37103989 & - \\
\hline $\mathrm{INc}_{1}$ & -4148.494740 & -4149.38834072 & - \\
\hline $\mathrm{INd}_{1}$ & -4148.488325 & -4149.38285858 & -301.96 \\
\hline $\mathrm{TSa}_{1-2}$ & -4148.472090 & -4149.36571944 & -307.60 \\
\hline $\mathrm{TSb}_{1-2}$ & -4148.465214 & -4149.35864060 & -294.23 \\
\hline $\mathrm{TSc}_{1-2}$ & -4148.483109 & -4149.37715400 & -317.33 \\
\hline $\mathrm{TSd}_{1-2}$ & -4148.472149 & -4149.36556012 & - \\
\hline $\mathrm{INa}_{2}$ & -4148.506967 & -4149.40246475 & - \\
\hline $\mathrm{INb}_{2}$ & -4148.520172 & -4149.41525876 & - \\
\hline $\mathrm{INc}_{2}$ & -4148.500447 & -4149.39720518 & - \\
\hline $\mathrm{INd}_{2}$ & -4148.523729 & -4149.41741396 & -480.43 \\
\hline $\mathrm{TSa}_{2-3}$ & -4148.488920 & -4149.38306691 & -468.69 \\
\hline $\mathrm{TSb}_{2-3}$ & -4148.497823 & -4149.39119515 & -482.50 \\
\hline $\mathrm{TSc}_{2-3}$ & -4148.490968 & -4149.38418714 & -464.90 \\
\hline $\mathrm{TSd}_{2-3}$ & -4148.496686 & -4149.38908128 & - \\
\hline $\mathrm{INa}_{3}$ & -4148.542003 & -4149.44003885 & - \\
\hline $\mathrm{INb}_{3}$ & -4148.555599 & -4149.45311744 & - \\
\hline $\mathrm{INc}_{3}$ & -4148.541451 & -4149.43881563 & - \\
\hline $\mathrm{INd}_{3}$ & -4148.555336 & -4149.45261744 & -109.63 \\
\hline $\mathrm{TSa}_{3-4}$ & -4224.971441 & -4225.89114117 & -50.33 \\
\hline $\mathrm{TSb}_{3-4}$ & -4224.975100 & -4225.89618673 & -88.47 \\
\hline $\mathrm{TSc}_{3-4}$ & -4224.976901 & -4225.89676103 & -90.32 \\
\hline $\mathrm{TSd}_{3-4}$ & -4224.968837 & -4225.88939314 & - \\
\hline $\mathrm{INa}_{4}$ & -4224.993713 & -4225.91268535 & - \\
\hline $\mathrm{INb}_{4}$ & -4225.010423 & -4225.92675275 & - \\
\hline $\mathrm{INc}_{4}$ & -4225.012698 & -4225.93120510 & - \\
\hline $\mathrm{INd}_{4}$ & -4225.007114 & -4225.92279562 & - \\
\hline prod & -848.598145 & -848.904304669 & - \\
\hline
\end{tabular}


Table S2. Free energies $(G)$, total electronic energies $(E)$, and the imaginary frequencies in solvent (tetrahydrofuran/water 10:1, v/v) for the optimized species for ARO reaction of $p$-methoxyphenylboronic acid $\mathbf{8 b}$ and $\mathbf{O A}$, as evaluated by DFT calculation (B3LYP25,26/def2SVP ${ }^{27} / S^{2} \mathrm{MD}^{29} / /$ B3LYP $25,26 /$ def2TZVP27/SMD ${ }^{29}$ ).<smiles>COCC1C=CC(c2ccc(OC)cc2)C(O)C1COC</smiles>

\begin{tabular}{|c|c|c|c|}
\hline Species & $G$ (hartree) & $E$ (hartree) & Imaginary Frequency $\left(\mathrm{cm}^{-1}\right)$ \\
\hline TMRh & -3646.764676 & -3647.44834388 & - \\
\hline TMRh-THF & -3879.184384 & -3879.98212949 & - \\
\hline $\mathrm{INa}_{1}$ & -4263.029732 & -4263.95462941 & - \\
\hline $\mathrm{INb}_{1}$ & -4263.016230 & -4263.94122848 & - \\
\hline $\mathrm{INc}_{1}$ & -4263.035997 & -4263.95784324 & - \\
\hline $\mathrm{INd}_{1}$ & -4263.029590 & -4263.95318443 & - \\
\hline $\mathrm{TSa}_{1-2}$ & -4263.010628 & -4263.93439674 & -300.07 \\
\hline $\mathrm{TSb}_{1-2}$ & -4263.005969 & -4263.92897239 & -305.19 \\
\hline $\mathrm{TSc}_{1-2}$ & -4263.024228 & -4263.94701703 & -294.90 \\
\hline $\mathrm{TSd}_{1-2}$ & -4263.013166 & -4263.93650936 & -317.76 \\
\hline $\mathrm{INa}_{2}$ & -4263.048594 & -4263.97477171 & - \\
\hline $\mathrm{INb}_{2}$ & -4263.063815 & -4263.98646415 & - \\
\hline $\mathrm{INc}_{2}$ & -4263.042932 & -4263.96874338 & - \\
\hline $\mathrm{INd}_{2}$ & -4263.065273 & -4263.99081829 & - \\
\hline $\mathrm{TSa}_{2-3}$ & -4263.030925 & -4263.95423390 & -479.72 \\
\hline $\mathrm{TSb}_{2-3}$ & -4263.038875 & -4263.96147393 & -467.74 \\
\hline $\mathrm{TSc}_{2-3}$ & -4263.033992 & -4263.95546063 & -481.82 \\
\hline $\mathbf{T S d}_{2-3}$ & -4263.038457 & -4263.95986164 & -462.66 \\
\hline $\mathrm{INa}_{3}$ & -4263.083989 & -4264.01115607 & - \\
\hline $\mathrm{INb}_{3}$ & -4263.097252 & -4264.02451051 & - \\
\hline $\mathrm{INc}_{3}$ & -4263.083976 & -4264.01033284 & - \\
\hline $\mathrm{INd}_{3}$ & -4263.097753 & -4264.02418241 & - \\
\hline $\mathrm{TSa}_{3-4}$ & -4339.511511 & -4340.45979083 & -108.47 \\
\hline $\mathrm{TSb}_{3-4}$ & -4339.516339 & -4340.46740089 & -80.14 \\
\hline $\mathrm{TSc}_{3-4}$ & -4339.519264 & -4340.46756647 & -101.92 \\
\hline $\mathrm{TSd}_{3-4}$ & -4339.512053 & -4340.46244817 & -61.10 \\
\hline $\mathrm{INa}_{4}$ & -4339.541714 & -4340.48938754 & - \\
\hline $\mathrm{INb}_{4}$ & -4339.554257 & -4340.49847878 & - \\
\hline $\mathrm{INc}_{4}$ & -4339.561682 & -4340.50603370 & - \\
\hline $\mathrm{INd}_{4}$ & -4339.548949 & -4340.49608669 & - \\
\hline prod & -963.143652 & -963.478707213 & - \\
\hline
\end{tabular}


Table S3. Free energies (G), total electronic energies (E), and the imaginary frequencies in solvent (tetrahydrofuran/water 10:1, v/v) for the optimized species for ARO reaction of $m$-iodophenylboronic acid 8c and OA, as evaluated by DFT calculation (B3LYP $25,26 /$ def2SVP ${ }^{27} / S^{2} D^{29} / /$ B3LYP ${ }^{25,26 / d e f 2 T Z V P 27 / S M D ~}{ }^{29}$ ).<smiles>COCC1C=CC(c2cccc(I)c2)C(O)C1COC</smiles>

\begin{tabular}{|c|c|c|c|}
\hline Species & $G$ (hartree) & $E$ (hartree) & Imaginary Frequency $\left(\mathrm{cm}^{-1}\right)$ \\
\hline TMRh & -3829.436333 & -3830.07664334 & - \\
\hline TMRh-THF & -4061.856806 & -4062.61208333 & - \\
\hline $\mathrm{INa}_{1}$ & -4445.703585 & -4446.58381747 & - \\
\hline $\mathrm{INb}_{1}$ & -4445.690464 & -4446.57192698 & - \\
\hline $\mathrm{INc}_{1}$ & -4445.708463 & -4446.58782476 & - \\
\hline $\mathrm{INd}_{1}$ & -4445.704229 & -4446.58411739 & - \\
\hline $\mathrm{TSa}_{1-2}$ & -4445.689406 & -4446.56961465 & -293.21 \\
\hline $\mathrm{TSb}_{1-2}$ & -4445.678178 & -4446.55743977 & -306.41 \\
\hline $\mathrm{TSc}_{1-2}$ & -4445.694872 & -4446.57464173 & -286.57 \\
\hline $\mathrm{TSd}_{1-2}$ & -4445.685765 & -4446.56450195 & -323.96 \\
\hline $\mathrm{INa}_{2}$ & -4445.721394 & -4446.60250812 & - \\
\hline $\mathrm{INb}_{2}$ & -4445.730234 & -4446.61129868 & - \\
\hline $\mathrm{INc}_{2}$ & -4445.712891 & -4446.59521479 & - \\
\hline $\mathrm{INd}_{2}$ & -4445.734755 & -4446.61598091 & - \\
\hline $\mathrm{TSa}_{2-3}$ & -4445.699010 & -4446.57920470 & -485.91 \\
\hline $\mathrm{TSb}_{2-3}$ & -4445.704675 & -4446.58441495 & -461.52 \\
\hline $\mathrm{TSc}_{2-3}$ & -4445.703464 & -4446.58163727 & -484.32 \\
\hline $\mathrm{TSd}_{2-3}$ & -4445.707768 & -4446.58642434 & -463.44 \\
\hline $\mathrm{INa}_{3}$ & -4445.752973 & -4446.63734656 & - \\
\hline $\mathrm{INb}_{3}$ & -4445.766883 & -4446.65069414 & - \\
\hline $\mathrm{INc}_{3}$ & -4445.754675 & -4446.63669682 & - \\
\hline $\mathrm{INd}_{3}$ & -4445.767462 & -4446.65018702 & - \\
\hline $\mathrm{TSa}_{3-4}$ & -4522.183463 & -4523.08883384 & -98.27 \\
\hline $\mathrm{TSb}_{3-4}$ & -4522.186896 & -4523.09353893 & -66.93 \\
\hline $\mathrm{TSc}_{3-4}$ & -4522.189783 & -4523.09408376 & -96.62 \\
\hline $\mathrm{TSd}_{3-4}$ & -4522.182773 & -4523.08854200 & -50.49 \\
\hline $\mathrm{INa}_{4}$ & -4522.216757 & -4523.11756404 & - \\
\hline $\mathrm{INb}_{4}$ & -4522.210096 & -4523.11498267 & - \\
\hline $\mathrm{INc}_{4}$ & -4522.225174 & -4523.12887064 & - \\
\hline $\mathrm{INd}_{4}$ & -4522.212548 & -4523.11716089 & - \\
\hline prod & -1145.819195 & -1146.11064472 & - \\
\hline
\end{tabular}

